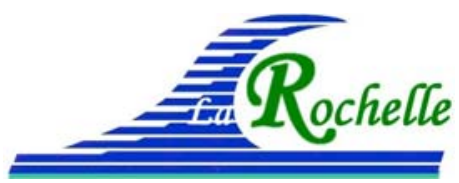

\title{
Grands brise-lames à écrans minces
}

\section{Jean BOUGIS ${ }^{1}$}

1. Cabinet Jean Bougis - Ingénieur Conseil - 32/34 chemin du Moulin 06650 Opio, France.

jean.bougis@wanadoo.fr

\section{Résumé :}

Les brise-lames partiels, qui offrent un passage libre à l'eau au-dessous d'une certaine cote, permettent de protéger des plans d'eau contre une agitation de longueur d'onde relative pas trop grande. Le concept de brise-lames partiels composés d'un écran mince suspendu à une estacade s'est d'abord développé dans l'ex-URSS au cours de la première moitié du $\mathrm{XX}^{\mathrm{e}}$ siècle, puis en occident. Des ouvrages de petites dimensions ont été construits en France. Plus récemment, deux grands brise-lames à écran mince on été construits au port du Château à Brest et au port des Minimes de La Rochelle. L'article présente successivement un bref historique de ce type d'ouvrages, leurs fondements théoriques, les éléments de dimensionnement (théoriques, numériques et expérimentaux), les exemples des ouvrages réalisés à Brest et à La Rochelle. Des essais en canal réalisés par l'auteur mettent en évidence le fonctionnement particulier de ces ouvrages pour de grandes longueurs d'ondes, ainsi que les dispositions constructives à adopter en particulier en termes de maîtrise des risques d'affouillements.

Mots clés : Brise-lames, Digues partielles, Ecrans minces, Essais en canal, Transmission, Réflexion, Dissipation, Efforts hydrodynamiques.

\begin{abstract}
:
Partial breakwaters, which left a free passage to water below some level, allow to protect free surface region against wave agitation of relatively small relative wavelengths. The concept of fixed breakwaters consisting of a thin barrier suspended from a wharf, first developed in the former USSR during the first half of the twentieth century, and then in the occident. Small structures have been built in France. More recently, two large thin-screen breakwaters have been constructed at the Port du Château in Brest and at the Port des Minimes in La Rochelle. This paper presents successively a brief history of this type of works, their theoretical bases, the dimensioning elements (theoretical, numerical and experimental), the examples of the works realized in Brest and La Rochelle. Wave flume tests carried out by the author highlight the particular functioning of these structures for long wavelengths, as well as the construction arrangements to be adopted in particular to control the scouring risks.
\end{abstract}

Keywords: Breakwater, Partial dikes, Thin screens, Wave flume tests, Transmission, Reflection, Dissipation, Hydrodynamic forces. 


\section{Thème 4 - Ouvrages portuaires, offshore et de plaisance}

\section{Introduction}

Les brise-lames partiels, qui offrent un passage libre à l'eau au-dessous d'une certaine cote, permettent de protéger des plans d'eau contre une agitation de longueur d'onde pas trop grande devant la profondeur d'eau. Ces brise-lames partiels prennent soit la forme d'un caisson prismatique de section rectangulaire supporté par des pieux, soit celle d'un écran mince suspendu à une estacade. Ce type d'ouvrages a été développé au cours de la première moitié du $\mathrm{XX}^{\mathrm{e}}$ siècle, d'abord dans l'ex-URSS (DJOUNKOVSKI \& BOJITCH 1959), puis en occident (DEAN \& HARLEMAN 1966). Depuis, de nombreuses études théoriques et expérimentales ont été menées et des ouvrages ont été construits en France. Les uns de petites dimensions (Charpignat sur le lac du Bourget dans les années 1980, Port-Servaux à Marseille en 2005, etc.), les autres de grandes dimensions (Port du Château à Brest en 2008, Port des Minimes à La Rochelle en 2014).

\section{Fondements théoriques}

Soit un repère orthonormé direct $O x y z$, dont l'origine $O$ est située sur la surface libre et sur l'écran, l'axe $O x$ est parallèle à l'écran, l'axe $O z$ est vertical ascendant et l'axe $O y$ est normal à l'écran et orienté dans le sens de la propagation de la houle incidente de période $T$, de pulsation $\omega(\omega T=2 \pi)$, de longueur d'onde $\lambda$, de nombre d'onde $k(k \lambda=2 \pi)$. La profondeur d'eau est notée $h$ et l'écran descend jusqu'à la cote $z=-d$.

\subsection{Approche énergétique}

Considérons le volume fluide constitué de l'ensemble des particules incluses, au repos, dans le domaine $D(d)$ délimité par :

- deux plans verticaux parallèles à la direction de propagation de la houle et distants d'un mètre ;

- deux plans verticaux perpendiculaires à la direction de propagation de la houle et distants d'une longueur d'onde $\lambda$;

- deux plans horizontaux de cotes respectives $z=-d$ et $z=0$.

L'énergie spécifique totale (potentielle et cinétique) de la houle $e_{h}(d)$ comprise entre la cote $z=-d$ et la surface libre s'écrit :

$e_{h}(d)=\frac{1}{8} \rho g H^{2}\left[1-\frac{\operatorname{sh} k(h-d)}{\operatorname{sh} k h} \frac{\operatorname{sh} k(2 h-d)}{\operatorname{sh} 2 k h}\right]$

La figure 1 présente la densité moyenne d'énergie de la houle véhiculée dans la tranche d'eau située au-dessus de la cote $z=-d$, pour différents nombres d'onde de la houle. S'il était possible d'arrêter toute l'énergie véhiculée par les particules d'eau situées au repos au-dessus d'une cote $z=-d$ donnée en laissant passer toute l'énergie véhiculée dans la tranche d'eau inférieure, le coefficient de transmission qui en résulterait serait donné par la formule empirique de Kondratief (DJOUNKOVSKI \& BOJITCH 1959) :

$C_{T}(d)=\sqrt{\frac{\operatorname{sh} k(h-d)}{\operatorname{sh} k h} \frac{\operatorname{sh} k(2 h-d)}{\operatorname{sh} 2 k h}} \quad ; \quad C_{R}(d)=\sqrt{1-C_{T}^{2}(d)}$ 


\section{XVèmes Journées Nationales Génie Côtier - Génie Civil \\ La Rochelle, 29 au 31 mai 2018}

donnée par son auteur pour des rapports $\lambda / h \leq 0.35$.

L'idée d'utiliser des brise-lames partiels est donc fondée sur le fait que, lorsque la profondeur relative $h / \lambda$ est suffisamment importante, la majeure partie de l'énergie de la houle est concentrée dans la partie supérieure de la veine fluide et qu'il est donc possible d'en limiter la transmission en interceptant ce flux d'énergie par un écran partiel.
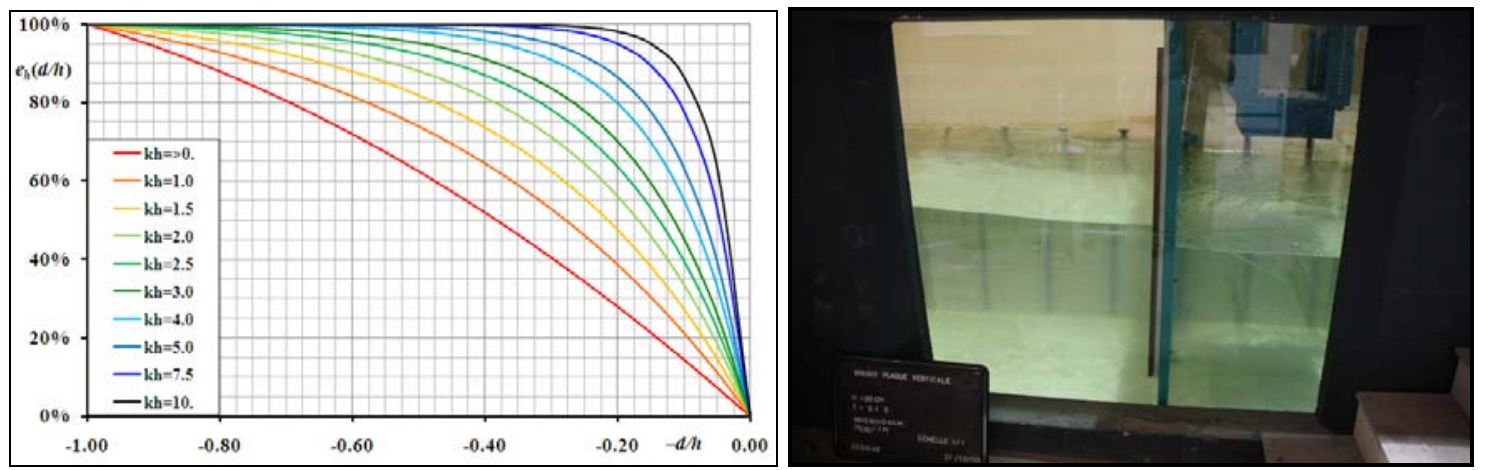

Figure 1. a) Répartition verticale de l'énergie de la houle - b) Essais en canal à ACRI.

\subsection{Approche analytique}

\subsubsection{Profondeur infinie}

URSELL (1947) a traité analytiquement le cas d'un voile vertical traversant la surface libre et immergé jusqu'à une profondeur donnée $d$ dans une veine fluide de profondeur infinie. Suite à des développements fondés sur une transformation intégrale sur le demiaxe vertical négatif, il a obtenu les coefficients de transmission et de réflexion suivants :

$$
C_{T}(d)=\frac{K_{1}\left(k_{0} d\right)}{\sqrt{\pi^{2} I_{1}^{2}\left(k_{0} d\right)+K_{1}^{2}\left(k_{0} d\right)}} \quad ; \quad C_{R}(d)=\frac{\pi I_{1}\left(k_{0} d\right)}{\sqrt{\pi^{2} I_{1}^{2}\left(k_{0} d\right)+K_{1}^{2}\left(k_{0} d\right)}}
$$

où $k_{0}$ est le nombre d'onde en grande profondeur et où $I_{1}$ et $K_{1}$ désignent respectivement les fonctions de Bessel modifiées de première et de deuxième espèce d'ordre 1 .

\subsubsection{Profondeur finie}

LOSADA et al. (1992) ont obtenu une solution analytique fondée sur le développement du potentiel des vitesses la base des fonctions propres en profondeur finie. Les coefficients des foncions du développement sont déterminés numériquement au sens des moindres carrés.

\subsection{Puissance transmise}

WIEGEL (1960) a développée une solution pour un écran vertical traversant la surface libre et immergé à la cote $z=-d$ dans une veine fluide de profondeur finie $h$. Son approche est fondée sur l'évaluation de la puissance moyenne transmise sous l'écran.

$C_{T}(d)=\sqrt{\frac{2 k(h-d)+\operatorname{sh} 2 k(h-d)}{2 k h+\operatorname{sh} 2 k h}} \quad ; \quad C_{R}(d)=\sqrt{1-\frac{2 k(h-d)+\operatorname{sh} 2 k(h-d)}{2 k h+\operatorname{sh} 2 k h}}$ 


\section{Thème 4 - Ouvrages portuaires, offshore et de plaisance}

La solution a été sensiblement améliorée pour les faibles longueurs d'onde par KRIEBEL \& BOLLMANN (1996) qui ont proposé la formulation suivante prenant en compte l'énergie de l'onde réfléchie :

$$
C_{T}(d)=\frac{2 T_{F}}{1+T_{F}} \quad ; \quad T_{F}=\frac{2 k(h-d)+\operatorname{sh} 2 k(h-d)}{2 k h+\operatorname{sh} 2 k h}
$$

\section{Dimensionnement numérique}

L'évaluation des performances d'un écran mince peut se faire numériquement au moyen d'un logiciel de diffraction-radiation bidimensionnel dans le plan vertical. A cet effet, l'auteur a développé une méthode d'équations intégrales hybride localisée au voisinage de la structure, entre les ordonnées $y=Y^{-}$et $y=Y^{+}$sur une longueur égale à quelques fois la profondeur et raccordée sur des développements en séries sur les fonctions propres du problème. On considère une houle incidente dont le potentiel des vitesses s'écrit :

$$
\phi_{I}=\mathfrak{R}\left\{\tilde{\phi}_{I} \exp (-i \omega t)\right\} \quad \text { avec } \quad \tilde{\phi}_{I}=-i A_{I} \frac{g}{\omega} \frac{\operatorname{ch} k(z+h)}{\operatorname{ch} k h} \exp (i k y)
$$

et on détermine un potentiel des vitesses $\widetilde{\phi}$ qui satisfait le problème aux limites suivant :

$$
\begin{array}{ll}
\Delta \widetilde{\phi}=0 & -h \leq z \leq 0 \\
\frac{\partial \widetilde{\phi}}{\partial z}-k_{0} \widetilde{\phi}=0 & z=0 \\
\frac{\partial \widetilde{\phi}}{\partial z}=0 & z=-h \\
\frac{\partial \widetilde{\phi}}{\partial n}=0 & \text { sur l'écran }
\end{array}
$$

et qui se raccorde aux expressions suivantes :

$$
\begin{array}{ll}
\widetilde{\phi}^{-}=-i \frac{g}{\omega} \frac{\operatorname{ch} k(z+h)}{\operatorname{ch} k h}\left[A_{I} \exp (+i k y)+A_{R} \exp (-i k y)\right]+\sum_{n=1}^{\infty} \frac{\cos k_{n}(z+h)}{\cos k_{n} h} A_{n}^{-} \exp \left(+k_{n} y\right) & y=Y^{-} \\
\widetilde{\phi}+=-i \frac{g}{\omega} \frac{\operatorname{ch} k(z+h)}{\operatorname{ch} k h} A_{T} \exp (+i k y)+\sum_{n=1}^{\infty} \frac{\cos k_{n}(z+h)}{\cos k_{n} h} A_{n}^{+} \exp \left(-k_{n} y\right) & y=Y^{+}
\end{array}
$$

où le nombre d'onde $k$ et les coefficients $k_{n}$ vérifient les équations de dispersions :

$$
k \text { th } k h=k_{0} \quad \text { et } \quad k_{n} \operatorname{tg} k_{n} h=-k_{0} \quad \text { avec } \quad k_{0}=\frac{\omega^{2}}{g}
$$

Les frontières du domaine de calcul supportent une distribution superficielle mixte de Green de singularités de Rankine. La surface libre, le fond et la structure sont discrétisés en éléments avec des singularités constantes; le potentiel des vitesses étant inconnu. Les frontières de raccord avec les développements analytiques supportent des singularités dont les fonctions de forme sont respectivement $\operatorname{ch} k(z+h)$ et $\cos k_{n}(z+h)$; les amplitudes $A_{R}, A_{T}, A_{n}{ }^{-}$et $A_{n}{ }^{+}$étant inconnues. La résolution du système fournit directement les amplitudes des champs de vagues des raccords. Le nombre de termes des développements varie de 1 à 5 selon le rapport entre la distance des frontières de raccord et la profondeur. L'intégration des pressions permet d'évaluer les efforts dus à la houle. 


\section{XVèmes Journées Nationales Génie Côtier - Génie Civil \\ La Rochelle, 29 au 31 mai 2018}

\section{Essais en canal à houle}

Des essais en canal à houle ont permis d'évaluer la transmission des écrans minces. COOPER \& LONGUET-HIGGINS (1951) ont mené des essais dans un canal en bois ( $9.70 \mathrm{~m}$ de long, $0.24 \mathrm{~m}$ de large et $0.50 \mathrm{~m}$ de haut) dans une profondeur de $0.415 \mathrm{~m}$ pour des longueurs d'onde de moins de $0.40 \mathrm{~m}: \lambda / \mathrm{h}<1$. Ils ont confirmé les calculs d'URSELL (1947) à 15\% près.

Pour valider son approche, WIEGEL (1960) a réalisé des essais dans le canal à houle de l'Université de Californie à Berkeley (60 ft de long, $1 \mathrm{ft}$ de large et $3 \mathrm{ft}$ de haut) dans des profondeurs de $1.53 \mathrm{ft}$ et $2.00 \mathrm{ft}$, pour des rapports $1.5<\lambda / h<5.9$.

KRIEBEL \& BOLLMANN (1996) rapportent des résultats d'essais qui ont été réalisés par l'U.S. Naval Academy Hydromecanics Laboritory (NAHL) et par la British Columbia Research Corporation (BCRC) pour des profondeurs relatives $1.3<\lambda / \mathrm{h}<5.6$.

Suite aux études d'exécution du projet du port du Château à Brest (2006 à 2007) pour lequel $\lambda / h \leq 4$, s'est profilé le projet du port des Minimes à La Rochelle (2009) en eau peu profonde avec $6.0<\lambda / \mathrm{h}<12$. Pour de tels $\lambda / \mathrm{h}$, la propagation de l'agitation sous le rideau s'accompagne d'une augmentation significative des vitesses horizontales, ce qui engendre trois nouvelles problématiques :

1) la diminution de la transmission due à la dissipation visqueuse ;

2) l'augmentation des efforts hydrodynamiques dus à la dissipation visqueuse ;

3) les risques d'affouillements du sol sablo-vaseux par le passage de l'eau sous l'écran.

Pour évaluer ces effets, l'auteur a fait réaliser, en novembre 2009, des essais en canal à houle au laboratoire d'ACRI-IN à Sophia-Antipolis (31.0 m de long, $1.8 \mathrm{~m}$ de large et $1.8 \mathrm{~m}$ de haut) dans une profondeur de $1.0 \mathrm{~m}$ et pour des longueurs d'ondes relatives variant de 0.33 à 10 et des écrans de profondeur $d / h=0.25,0.50,0.75$ et 0.90 . Deux hauteurs de houle ont été testées, l'une $H \approx 0.2 \mathrm{~m}$ et l'autre de la moitié (ACRI-IN 2009).

Des essais ont été réalisés dans un canal du Nanjing Hydraulic Research Institute en Chine (64.0 m de long, $1.8 \mathrm{~m}$ de large et $1.8 \mathrm{~m}$ de haut) dans une profondeur de $0.60 \mathrm{~m}$, avec $2.0<\lambda / h<12.25$, pour des écrans de profondeur $d / h$ de $0.1,0.2,0.3,0.5$ et 0.8 et pour des hauteurs $H / d$ de $0.1,0.2,0.3$ et 0.4 (LIEHONG et al. 2010).

\section{Transmission des écrans}

La figure 2 présente, en fonction du rapport $\lambda / \mathrm{h}$, le coefficient de transmission $C_{T}$ des écrans minces de tirant d'eau variable d'après les différentes sources. Les essais de LIEHONG et al. (2010) ayant été réalisés pour des rapports $d / h$ de $0.1,0.2,0.3,0.5$ et 0.8 . Les valeurs indiquées pour $0.25,0.75$ et 0.90 ont donc été interpolées.

On note que les résultats théoriques sont répartis en deux groupes cohérents : un groupe de solutions analytiques et numériques et un groupe de solutions énergétiques.

Pour $\lambda / h<3$ ou 4, les résultats expérimentaux sont en bon accord avec les solutions théoriques et avec la solution de KRIEBEL et BOLLMANN (1996), tandis que les deux autres solutions énergétiques en sont plus éloignées. 


\section{Thème 4 - Ouvrages portuaires, offshore et de plaisance}

Pour $\lambda / h>4$ ou 5 , les résultats expérimentaux se situent entre les deux groupes théoriques et énergétiques, en se rapprochant des courbes énergétiques au fur et à mesure que le rapport $d / h$ augmente.
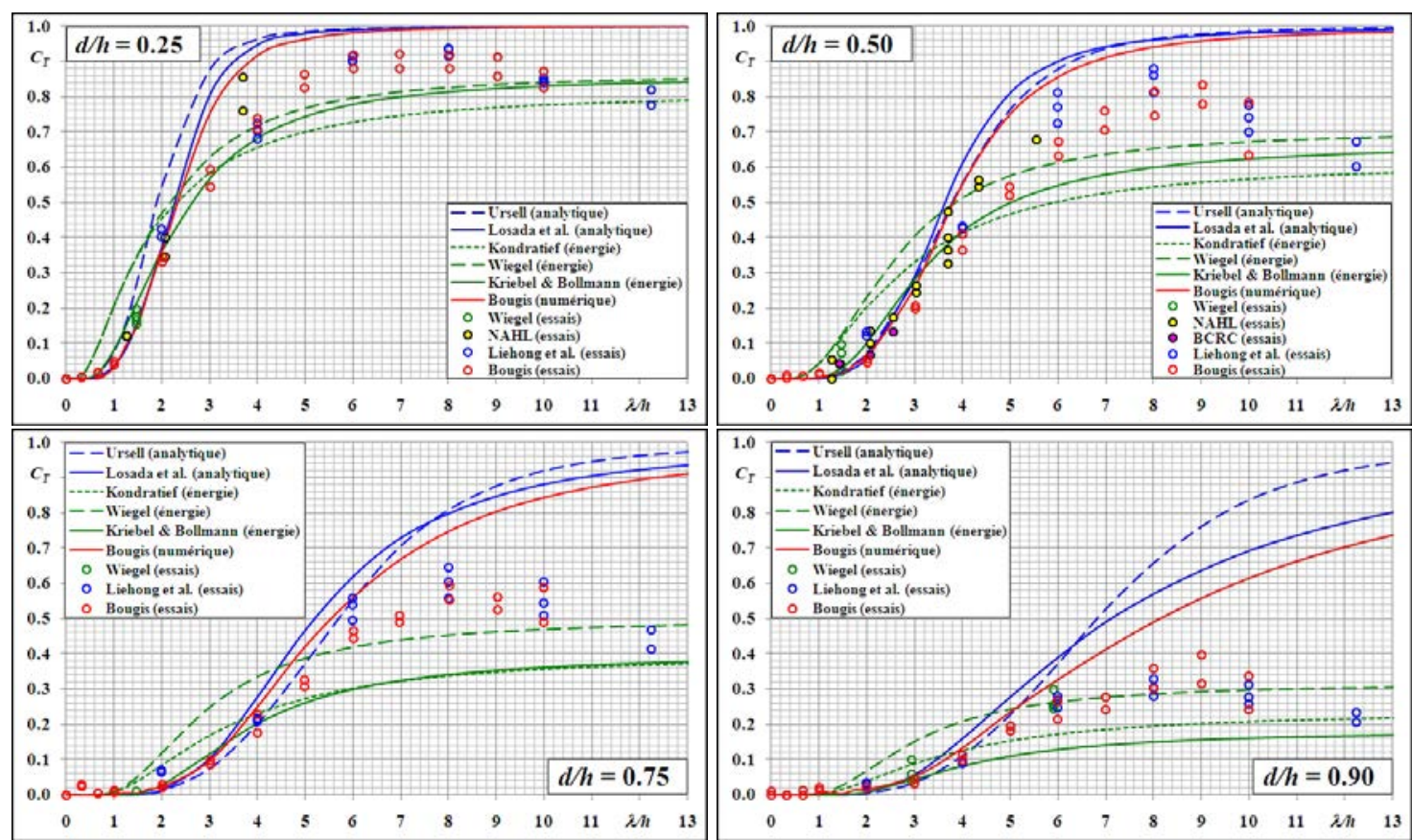

Figure 2. Coefficient de transmission selon les différentes sources.

Le fait que le coefficient de transmission expérimental passe par un maximum lorsque $\lambda / h$ est de l'ordre de 6 à 10, puis diminue ensuite est remarquable. Ce phénomène, n'est pas explicable par la théorie du potentiel qui sous-tend les solutions analytiques et numériques. Compte tenu que la réflexion tend vers zéro quand la longueur d'onde croît, son origine doit être recherchée dans la dissipation visqueuse et tourbillonnaire à la base de l'écran. En effet, pour une hauteur $H$ donnée, la vitesse horizontale sous l'écran augmente avec la longueur d'onde jusqu'à une valeur limite ; ce qui engendre une dissipation visqueuse et tourbillonnaire importante qui prélève de l'énergie sur la transmission qui doit donc diminuer avant de se stabiliser quand la puissance véhiculée par la houle et la dissipation de l'écran mince tendent vers leur valeurs limites respectives. Par ailleurs, comme la puissance transmise par la houle est proportionnelle à $H^{2}$ tandis que la dissipation visqueuse est proportionnelle à $H^{3}$, le coefficient de transmission décroît avec la hauteur $H$ de la houle ; phénomène clairement mis en évidence dans les essais.

\section{Exemples de grands ouvrages}

\subsection{Le port du Château à Brest}

L'aménagement du port du Château a été réalisé en 2008 par le groupement Quille (Groupe Bouygues) et ETPO. Cette protection, située dans la rade Abri, est composée 


\section{XVèmes Journées Nationales Génie Côtier - Génie Civil \\ La Rochelle, 29 au 31 mai 2018}

de deux ouvrages : au Sud une digue de $465 \mathrm{~m}$ de long (ouvrage de raccordement et 51 travées de $9.0 \mathrm{~m}$ ) et à l'Ouest une contre digue de $171 \mathrm{~m}$ de long (ouvrage de raccordement et 17 travées de $9.0 \mathrm{~m}$ ).

Les digues sont constituées d'un écran partiel, entre les cotes $-3.75 \mathrm{~m} \mathrm{CM}$ et $+9.70 \mathrm{~m}$ $\mathrm{CM}$, supporté par une estacade de $7.45 \mathrm{~m}$ de large fondées sur des portiques bi-pieux de $6.0 \mathrm{~m}$ d'entraxe, ancrés dans le substratum rocheux et munis de tirants précontraints. Le substratum est recouvert d'une couche de vase d'environ $8 \mathrm{~m}$ d'épaisseur. La digue Sud est construite dans une profondeur d'eau allant de $5.0 \mathrm{~m}$ CM à sa racine jusqu'à $8.0 \mathrm{~m}$ $\mathrm{CM}$ à son musoir ; la digue Ouest est construite dans une profondeur d'eau allant de 2.5 $\mathrm{m} \mathrm{CM}$ à sa racine jusqu'à $6.0 \mathrm{~m} \mathrm{CM}$ à son musoir. Le marnage est de $8.2 \mathrm{~m}$.

Les états de mer de projet sont respectivement au Sud et à l'Ouest :

- 1 an $H_{s}=0.7 \mathrm{~m} T_{p}=3.9 \mathrm{~s} ; 10$ ans $H_{s}=0.9 \mathrm{~m} T_{p}=4.3 \mathrm{~s} ; 100$ ans $H_{s}=1.1 \mathrm{~m} T_{p}=4.6 \mathrm{~s}$.

- 1 an $H_{s}=0.7 \mathrm{~m} T_{p}=3.1 \mathrm{~s} ; 10$ ans $H_{\mathrm{s}}=0.9 \mathrm{~m} T_{p}=3.6 \mathrm{~s} ; 100$ ans $H_{s}=1.1 \mathrm{~m} T_{p}=4.1 \mathrm{~s}$.

Les longueurs d'ondes relatives vérifient $1.0<\lambda / h<4.0$.

L'agitation résiduelle maximale est de $0.30 \mathrm{~m}$ pour les conditions annuelles et de 0.50 $\mathrm{m}$ pour les conditions décennales.
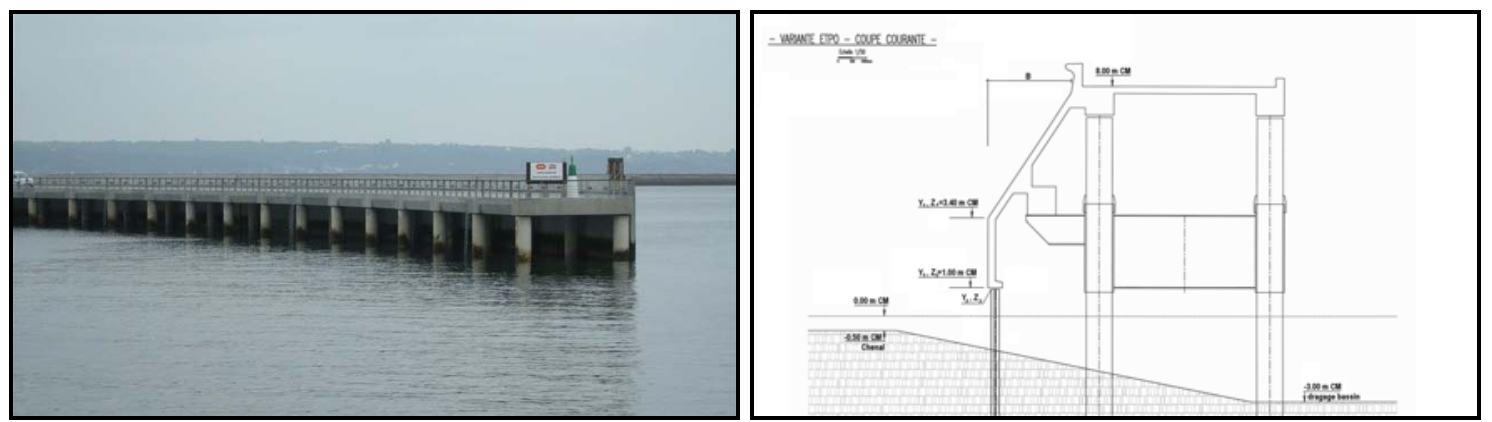

Figure 3. a) Digue Sud à Brest (ETPO) - b) Coupe de la digue de La Rochelle (ETPO).

\subsection{Le Port des Minimes à la Rochelle}

L'extension du port des Minimes a été achevée en 2014. La construction de la digue Nord a été réalisée par le groupement ETPO et SEMEN TP (enrochements). La digue de $624 \mathrm{~m}$ de long (tympans de fermeture et 68 travées de $9.0 \mathrm{~m}$ ) est raccordée au NordEst à la digue des Tamaris par une passerelle métallique de $156 \mathrm{~m}$ de long.

La digue est constituée d'un écran partiel supporté par une estacade de $9.5 \mathrm{~m}$ de large fondées sur des portiques bi-pieux de $6.0 \mathrm{~m}$ d'entraxe, foncés dans le sol sablo-vaseux qui est sub-affleurant à marée basse. Le marnage est de $8.1 \mathrm{~m}$.

L'écran mince est formé de deux "panneaux" plans surmontés d'un mur chasse mer. Le panneau du bas est vertical entre les cotes $+1.0 \mathrm{~m} \mathrm{CM}$ et $3.4 \mathrm{~m} \mathrm{CM}$. Le panneau du haut est incliné à $3 \mathrm{~V} / 2 \mathrm{H}$ vers l'intérieur du bassin.

Pour obtenir la protection du plan d'eau demandée, l'écran doit descendre jusqu'au sol. Compte tenu des risques d'affouillements du fond sablo-vaseux par le laminage de l'eau sous l'écran, deux solutions ont été envisagées : 


\section{Thème 4 - Ouvrages portuaires, offshore et de plaisance}

- disposer, sous le rideau, un tapis anti-affouillements en rip-rap, des gabions ou des matelas de berlingots de béton et un filtre en géotextile destiné à contenir les sédiments fins de la vase ;

- arrêter l'écran au-dessus du fond à une cote de l'ordre de $+1.0 \mathrm{~m} \mathrm{CM}$ et foncer un rideau vertical en palplanches arasé à $10 \mathrm{~cm}$ au-dessous du bas de l'écran (voir figure $3 \mathrm{~b}$ ).

Le critère de choix étant lié à l'espace maximal acceptable entre l'écran et l'antiaffouillement, qui a été fixé à $10 \mathrm{~cm}$ pour ne pas transmettre l'agitation, c'est la seconde solution qui a été retenue pour des raisons de fiabilité d'exécution et de durée de vie.

Les états de mer de projet sont : 1 an $H_{s}=1.3 \mathrm{~m} T_{p}=5.0 \mathrm{~s} ; 10$ ans $H_{s}=1.3 \mathrm{~m} T_{p}=6.0 \mathrm{~s}$; 100 ans $H_{s}=1.4 \mathrm{~m} T_{p}=7.5 \mathrm{~s}$. Les longueurs d'ondes relatives vérifient $6 .<\lambda / \mathrm{h}<12$.

La digue munie de son rideau de palplanche n'étant pas transparente à la houle, d'une part, seul son contournement contribue à l'agitation résiduelle dans les bassins du port et, d'autre part, les efforts hydrodynamiques sont ceux qui s'appliquent sur un mur.

\section{Références bibliographiques}

ACRI-IN. (2009). Comportement d'une plaque vertical semi-immergée. Rapport d'essais en canal à houle $n^{\circ}$ IN 547-794 R1 Ind0.

COOPER R. J. B., LONGUET-HIGGINS M. S. (1951). An experimental study of pressure variations in standing waves. Proceeding of Royal Society of London, vol. 206 A, p. 425-435.

DEAN R.G., HARLEMAN D. (1966). Interaction of Structures and Waves. IPPEN A., Ed., Estuary and Coastline Hydrodynamics, Chapitre 8, p. 341-406, New York: McGraw-Hill Book Company, INC.

DJOUNKOVSKI N.N., BOJITCH P.K. (1959). La houle et son action sur les côtes et les ouvrages côtiers, 404 p., Paris : Eyrolles.

KRIEBEL D.L., BOLLMANN C.A. (1996). Wave transmission past vertical wave barriers. Proceeding of $25^{\text {th }}$ International Conference of Coastal Engineering, ASCE, Orlando, Florida, 2-6 September, p. 2470-2483.

LIEHONG J., LI P., YANG J. H. (2010). Experimental research on coefficient of wave transmission through immersed vertical barrier of open-type breakwater. Proceeding of $32^{\text {th }}$ International Conference of Coastal Engineering, ASCE, Shanghai, China, 30 June -5 July, $13 \mathrm{p}$.

LOSADA I.J., LOSADA M.A., ROLDAN A.J. (1992) Propagation of oblique incident waves past a rigid vertical thin barriers, Applied Ocean Research, n¹4, p.191.199.

URSELL F. (1947) The effect of a fixed vertical barrier on surface waves in deep water, Proceedings of the Cambridge Philosophy Society, Vol. 43, p. 374-382. https://doi.org/10.1017/S0305004100023604

WIEGEL R. L. (1960) Transmission of waves past a rigid vertical thin barrier, ASCE, Journal of the waterways and Harbors Division, vol. 86, n²413, p. 1-12, March. 